\title{
Research on Development Orientation of Costume Design and
}

\section{Engineering}

\author{
Yanling $\mathrm{Hu}^{1}$ \\ ${ }^{1}$ Jiangxi Institute of Fashion Technology, Nanchang Jiangxi, 330201
}

KEYWORDS: Costume Design, Engineering, Development Orientation, Research

\begin{abstract}
Chinese apparel industry has experienced more than two decades of precipitation and accumulation of years of baptism to the Chinese clothing has brought a huge change. Chinese clothing experienced a shift from a commercial brand to clothing apparel manufacturers, derived from the Chinese clothing brand business, to refine the Chinese clothing, broken down into the changing nature of private label phase. This is a historic leap forward for Chinese clothing, the clothing just because China only twenty years time traveled overseas garment industry spent nearly a hundred years before the road traveled clothing. Over time, for the clothing industry development constraints increasingly blame, society, the mass of the garment industry increasingly high demand, China's garment industry is facing enormous challenges, the university of clothing design and engineering development orientation Education also need to find new export markets and industries to adapt to fashion design and development orientation of professional engineering requirements.
\end{abstract}

\section{Have a Clear Planning and Positioning on Individual Learning Career and Chosen Costume Design and Engineering}

Establish clear objectives like navigation lights on the road ahead, to illuminate the road ahead, not get lost, detours. Individual learning and career chosen costume design and engineering have a clear planning and positioning must be realistic, this is an important prerequisite, by understanding and evaluation of the individual to find personal strengths and weaknesses, based on individual learning ability, personal expertise found for personal professional goals and career planning, in order to make accurate and correct positioning of the individual learning plans and career as well as costume design and engineering.

Learning is boring, boring, often caused by human fatigue, learning a long time, it is easier to want to give, and often encounter difficulties and learning problems, mood will not smooth, so many people do not stick with it finally. Develop a clear and correct costume design and engineering study plan as if there were a conviction in the hearts of people, when faced with difficulties in learning or feel boring, beliefs give people support, giving strength, people insist down. Develop a clear and correct costume design and engineering study plan, in fact, represent a positive sense, this clothing design and engineering study has a good role in promoting. Prerequisite for success is good planning learning, beliefs and actively develop upward. Want to learn fashion design and engineering, we must do a good job planning, personal clothing design and engineering study have clear and unambiguous position, so-called "go rather fixed, and the world beyond into the matter," he says is the truth.

People most likely to commit a mistake to get carried away, not knowing self-evident, there is no clear understanding of the individual, it is often too bombastic or too timid, both of which are 
undesirable for personal development and learning has great drawbacks. Learn the most taboo thing is not realistic, which future learning and professional development laid a dangerous primer. Self-evaluation process is actually their own understanding and discovery process, normally very few people to make their own assessment, gradually ignored the individual's strengths and weaknesses, or perhaps engaged in learning is not good at personal aspects. This is also a realistic self-evaluation clothing design and engineering study in an important part, and only a clear understanding of individual strengths and weaknesses, to help individuals in favor of fashion design and engineering study and future career development planning to make accurate positioning, development personal good.

The main purpose of an assessment of the personal clothing design and engineering career for a variety of environmental assessment of the impact of career development, then a careful analysis of the causes for the results of the assessment of such results come out, and to find a solution Method. This is the future of personal clothing design and engineering career development has a positive effect, but also through personal clothing design and engineering assessment of the individual's career development after a clear positioning and understanding. Environment is one that everyone can not do without living conditions, so accurate personal clothing design and engineering to make a career assessment and planning, you need to analyze the individual characteristics of the environmental conditions in which to learn, change and development environment cases, the cause of the environment changes, the relationship between individuals and their environment, the environment, individual needs for personal development and learning requirements of environmental conditions, etc., sufficient to make an accurate assessment of the above environmental factors, in order to draw useful findings and then make a good plan for personal clothing design and engineering careers.

Personal clothing design and engineering career direction to make accurate positioning is based on realistic self-evaluation. Through self-evaluation, a clear understanding of individual strengths and weaknesses, and then combined with personal clothing design and engineering career planning and aspirations, can initially locate personal clothing design and engineering career direction, and then considering the individual aspects of good, avoid open personal weaknesses, and ultimately the direction of the professional career of personal clothing design and engineering to make accurate positioning.

The goal is the end result desired personal development, but also the great power of personal advancement. Core costume design and engineering development is targeted for future personal career development, career development need to set goals. Target is a lighthouse, lighthouse to light, can bring light to people on their way, will not get lost. Personal clothing design and engineering to set development goals will take time to accumulate, through long-term costume design and engineering study, careful consideration of individual ability in clothing design and engineering of the learning process, personal clothing design and engineering in the learning process the learning and personal expectations for the future in fashion design and engineering aspects, and establish individual professional costume design and engineering aspects of the short-term development goals, medium-term development objectives and long-term development goals, and then refine each target, broken down to individual behavior in a day, broken down into each stage of the target, and then one by one to achieve the final completion of the individual set development goals.

Setting goals, a good program, even if perfect, this is only part of paper, we need to put into action in order to truly achieve their goals. Goal is to command action after targeted according to action program implementation plans. Concrete measures personal clothing design and engineering operations from the following aspects: 
The fashion design and engineering process of learning, not only to teach fashion design and engineering expertise, skills, but also to grasp the popularity of costume design and engineering related professional experience and interview skills.

The costume design and engineering skills as the key content, the main purpose is to train individual practical ability.

The infiltration professionalism in fashion design and engineering studies, the training of individuals working ability.

Plan changes, so the needs for personal clothing design and engineering career is for continuous evaluation and feedback. Its amendments include: professional re-selection, career route choice, correcting life goals, implementation measures and program changes, and so on.

\section{Proposals for Development Orientation of Personal Clothing Design and Engineering}

Learning is a long way to go to do in personal and professional development orientation costume design project, the first personal need for clear and correct personal clothing design and engineering study planning, personal clothing design and engineering learning clear positioning; followed by the need realistic self-assessment to help you further develop more detailed, clearer personal clothing design and engineering professional learning plan, and this personal fashion design and engineering development orientation of great help; and can clearly know personally after the clothing design and engineering professional learning expectations, wants to achieve future career out of what the height and width of what, establish personal fashion design and engineering development goals, and pay all action, step by step, practical take every step, and finally reach personal expectations and achieve personal goals.

\section{References}

[1] Li Jun, Zhang Wenbin, Li Min, Yu Guoxing, Liuguan Bin. clothing design and engineering Research and Training targeting Course System [J]. textile education, 2006,06: 26-31.

[2] Liu Guanbin. clothing design and engineering development orientation [J]. Hunan Institute of Engineering (Social Science Edition), 2009,02: 98-101.

[3] Yang Mei. Garment Design and Engineering Training objectives of positioning [J] Chinese urban economy, 2011,20: 135.

[4] Liang Yalin, Zhang Xing, Xu Qingqing, Li Shuowen. in the 21st Century Fashion Design and Engineering Development Strategy [J]. Xi'an University Engineering Science and Technology, 2002,04: 315-317.

[5] Huang Dingqi. explore Garment Design and Engineering Practice Teaching Reform in the clothing industry development background [J]. Northern Literature (second half), 2012,08: 73. 